\title{
Designing Self-Aware Adaptive Systems: from Autonomic Computing to Cognitive Immune Networks
}

\author{
Nicola Capodieci \\ Università di Modena e Reggio Emilia \\ Modena, Italy
}

\author{
Emma Hart \\ Edinburgh Napier University \\ Edinburgh, UK
}

\author{
Giacomo Cabri \\ Università di Modena e Reggio Emilia \\ Modena, Italy
}

\begin{abstract}
An autonomic system is composed of ensembles of heterogeneous autonomic components in which large sets of components are dynamically added and removed. Nodes within such an ensemble should cooperate to achieve system or human goals, and systems are expected to self-adapt with little or no human-interaction. Designing such systems poses significant challenges. In this paper we propose that the system engineer might gain significant inspiration by looking to the biological immune system, particularly by adopting a perspective on the immune system proposed by Cohen known as the Cognitive Immune Network. The goal of this paper is to show how the current literature in autonomic computing could be positively enriched by considering alternative design processes based on cognitive immune networks. After sketching out the mapping in commonalities between the Cognitive Immune Network and the autonomic computing reference model, we demonstrate how these considerations regarding the design process can be exploited with an engineered autonomic system by describing experiments with a simple robotic swarm scenario.
\end{abstract}

Keywords-Awareness; Autonomic Computing; Artificial Immune Systems

\section{INTRODUCTION}

The current trend in designing cooperating entities is to think of them as acting as an ensemble, thus deploying autonomous features that enable the whole system to adapt to non-deterministic changes of the surrounding environment. Self-Adaptation (the ability to reach the optimal configuration according to external variables, achieved without human interaction) is strived for in situations in which it is too risky and/or too expensive to have a user provide incremental updates to hardware or software components of subsets of the ensemble. Example application scenarios include distributed or swarm robotics in disaster response scenarios [1] and homeland security [2] (so, safety-critical operations). In some contexts, if no-self-adaptation occurs, then constant monitoring or continuous user inputs would be necessary: pervasive service composition [3] and Multi-Agent System with Smart Grid integration [4] are cited just to name few applications. IBM's vision of autonomic computing [5] addresses some challenges related to this kind of applications. In particular, it focusses on robustness and reliability in distributed systems by introducing the concept of Self-Healing and the more general Self-Organization.

From a design perspective, mechanisms to achieve self-* properties must be able to deal with open and non-deterministic environments, and deal with a distributed notion of learning and reasoning. For this reason, designers have often turned to biology for inspiration. One biological system that has received much attention is the natural immune system: in a computational guise, Artificial Immune Systems (AIS) have been extensively used for a wide variety of applications in computing [6] from robotics to Internet security. From a biological perspective, a number of different theories exist to explain immune function. From an engineering perspective, this simply offers the engineer a rich source of material from which to take inspiration. In this article, we focus on one particular paradigm, that of the Cognitive Immune Network proposed in [7], as it links adaptation with selfawareness, thereby providing an interesting view on how to exploit alternative design steps for artificial applications of immunology. The aim of this paper is therefore to highlight connections and commonalities between autonomic computing studies for analysing adaptivity in ensembles of autonomous components and the cognition- and awareness-related aspects that emerge from immune networks, thus suggesting directions for future research. More specifically, IBM's framework for adaptation, synthesized with the MAPE-K feedback loop [8], will be integrated to the findings of Cohen [7], Orosz [9] and Stepney [10] regarding emergent implications of designing systems based on immune networks.

\section{RELATED WORK}

Previous work [11], [12], [13] outlined how the concept of awareness can be divided into categories so to provide taxonomies regarding different levels of awareness. From these taxonomies, we adopt a working definition of awareness as the property of a system that demonstrates cognition and learning.

With regard to adaptivity, in the literature many researchers have tried to provide formal definitions of it in order to be able to recognise adaptive and/or self-adaptive features in the design process of computational units. Zhu et al. in [14], define adaptivity in humans as the property to sustain themselves in dynamic environments - however, if we apply this definition to a computational problem so as to use software agents or robots instead of humans, we immediately have to face a challenge concerning defining or modelling the environment. Bruni et al., [15], tried to leave out the word environment from their definition of adaptivity. They proposed a framework for designing adaptive software components starting from a code perspective in which the program flow is composed of 
data and control, with the latter one specifying behaviours and interactions. A subset of data (as program variables) is then tagged as control data, and thus adaptation is defined as the run-time modification of this control data. By accepting this definition, the concept of adaptation strongly relies on subjectivity, therefore more case specific considerations are needed in order to have a full understanding. Instead of trying to provide formal definitions for adaptivity, we try to establish a connection between awareness features and the potential adaptive capabilities of one or more components by determining the factors that provide adaptation and cognition in artificial immune systems, and then see where these features can be positioned in a widely accepted notation for adaptation framework (the IBM's MAPE-K control loop [8]).

\section{FROM AWARENESS TO ADAPTIVITY}

In this section we analyse the "ingredients" of adaptivity, i.e. we take two important paradigms for building Self-* systems in order to extract and understand what makes them able to deploy adaptivity, taking into account that we are dealing in distributed cooperative scenarios. The first approach to be analysed (autonomic computing) is an evolution of maintenance cycles of standard computing scenarios, while the second one is a bio-inspired approach with emerging aware and cognitive features. We highlight differences and commonalities to then show how merging features from both approaches has the potential to be useful in future research regarding Self-* systems.

\section{A. Autonomic Computing and Ensembles}

Several approaches have been proposed as design methodologies and blueprints for building self-adaptive systems. Despite significant differences among all these approaches, one common factor is the requirement to incorporate a feedback loop, whether the loop exists inside or outside the logic of the single component [16].

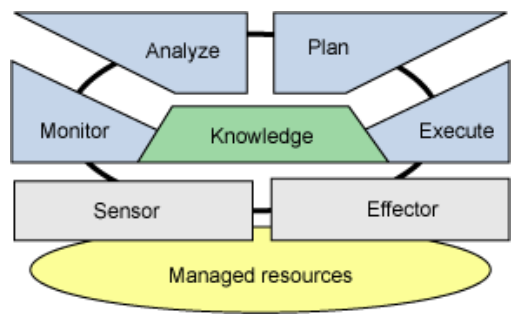

Fig. 1. MAPE-K for defining the autonomic management loop as introduced in [8]. Picture from http://www.ibm.com/developerworks/library/acitito/index.html

A widely accepted representation of a feedback loop is the one proposed by IBM in [8] and known as MAPE-K control loop (Fig. 1). The name MAPE-K summarizes the important phases that form the feedback loop.

- The (M) monitor phase deals with collection of everything that can be sensed from the environment; this data will be mainly collected through sensors.

- $\quad$ The analyze (A) phase is the first level of reasoning about the previously collected data.
- The plan (P) phase links the output of the previous phase with policies in order to elaborate a schedule regarding the next actions to take.

- The execute (E) phase is responsible with actually enforcing the previously constructed plan of actions: effectors or actuators will actually be used for interfacing the component with the managed resource.

Adaptivity lies in how the phases are connected to each other: cyclically, each executed plan will be evaluated thus providing a positive or a negative feedback that will reinforce or suppress the previously scheduled plan of actions. The history regarding executed plans and retrieved payoffs is retained in the knowledge repository $(\mathrm{K})$, so as to build a model able to learn from previous experiences. The element environment also plays a fundamental role: basically in this situation it may be defined as everything that can be directly or indirectly perceived (modified) through sensors (effectors). This model gets as more complex as we think of it as part of an ensemble: if this control loop is external to the single components, the presence of an autonomic manager that takes care of the MAPE phases is mandatory. More autonomic managers can be added to create hierarchies of managers: this can potentially represent a notable design issue since components can be unexpectedly added and removed or the whole system can be ideally divided according to task allocation policies, thus risking to have a non-optimal configuration of the autonomic managers. The complexity of dealing with these situations implies that the designer considers taking inspiration from other methodologies as well.

When trying to obtain self-adaptivity in an ensemble of components, another way is to think of the MAPE-K loop being embedded in the logic of every component. Awareness in this specific case plays a fundamental role: the constantly updated knowledge repository inside the single component makes the computational unit aware of its previous experiences, but in a distributed environment (the ensemble) these experiences should be shared among at least subsets of the ensemble so to create some sort of distributed knowledge. Distributed knowledge and experiences for cooperative behaviours need to be studied under a group-aware approach: this is a nontrivial issue that we try to address in the next section by following an alternative approach in designing ensembles. As for this section, it is important to stress that approaches related to Case-Based Reasoning (CBR) are commonly used for achieving adaptivity. It is trivial to understand that the knowledge repository in the MAPE-K control loop is perfectly complaint with the notion of cases in CBR; moreover, in idiotypic networks [17] (the kind of immune network exploited in the next section) some previous work has been done in order to show how this immune approach is inherently a variation of CBR [18].

\section{B. Awareness and Cognitive Aspects in Immune Networks}

In [19], Cohen defines a cognitive system as one that integrates three attributes: (i) it creates internal images of the world within which it exists; (ii) it self-organises by using experience to build and update its internal images; (iii) it is able to make decisions by choosing among alternatives. 
According to [19], these elements of cognition are central to the strategy of the immune system, which overall consists of three parts: recognition, i.e. sense signals and respond to them; cognition, i.e. interpret input signals, evaluate results and make decisions and finally, action, i.e. execute decisions. In this cognitive view, the immune system is regarded as a system that provides services of maintenance to the functioning body, a role broader than, but still covering, protection - the latter being the usually assumed function of the immune system.

Cohen maps functions observed in the natural immune system to this definition as follows:

- $\quad$ Creating images of the individual's internal and external environments is performed by the immune system at various levels: concrete, immune receptors provide physical negative images of the ligands they bind; abstract, interacting entities form process images which, for instance, may delineate a certain process of immune reaction; distributed, patterns of various immune molecules or cells exist throughout the body.

- Immune self-organisation is achieved by the progressive creation of information via learning and memory that happens across two scales: the innate germline history of the species, and the adaptive somatic experience of the individual.

- Decision- making is determined by the interactions of immune agents, the molecular dialogue that associates somatic perceptions with germ-line classes of behaviour in order to choose a particular type of response.

Cohen's view on the immune system is summarised in Fig. 2: an internal image is created through interaction of signals from the environment with entities in the system, generated from inherited (innate) components and components that have adapted due to individual experiences (the adaptive immune system). Decision making occurs by associating an image of the current environment, perceived through the receptor repertoire, with an appropriate response via a process known as co-respondence, in which agents react not only to their perception of the environment but to their perception of how other agents are responding. This is then executed as an inflammatory response.

It is relatively straightforward to map Cohen's cognitive view of the immune system to the MAPE-K model introduced earlier. A proposed mapping is shown in TABLE I. The reader's attention is drawn to two further points. The first regards the granularity of the mapping; in Cohen's immune network, the system or ensemble consists of a collection of cells that interact to provide adaptivity. In the context of an ensemble of autonomic components however it is more logical to think of each component in the ensemble containing a network of "cells"; this network gives rise to decisionmaking in the individual component. Components additionally share information, resulting in a second network based on topological relations between the components, and this results in a two-tiered architecture through which a global image repertoire emerges. Secondly, the role of the environment is key. A system (biological or computational) is defined as embodied if a feedback loop exists between a system and an

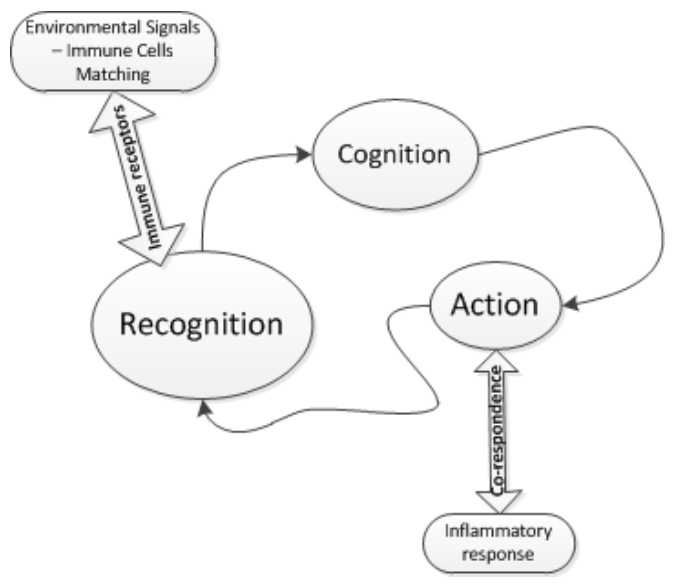

Fig. 2. Summarizing view on Cohen's cognitive immune network

environment such as the system is able to sense changes in the environment and it is able to provide subsequent responses by changing parts of the same environment according to the previously sensed information [10]. This implies the presence of perturbatory channels between the system and the environment - this is easily linked to the sensors and effectors introduced in the MAPE-K methodology.

Having made a tentative link between the MAPE-K control loop and Cohen's immune network model, we now go on to examine the emergent properties of such networks, in order to fully understand the implications for the design of computational self-adaptive systems.

\section{Emerging Properties for Fostering Self-Adaptation in Im- mune Networks}

In [9] the author describes four important principles of immune networks that lead to emergent properties, briefly summarized here.

1) Phylogenic Layering: this refers to the fact the newer images of responses are built on the top of older (and therefore pre-existing) inflammatory responses. Although trivial to understand, this principle has major implications, particularly in the context of autonomic computing. Firstly, it implies that at least some previously-exploited action plans should remain in some kind of system memory when they are replaced by a newer plan; crucially, the system responses to the monitored environmental situation must contain at least some components that can be re-used in future responses if layering is to be achieved. Finally, there should be a set of possible responses available to the Planning phase of the MAPE-K control loop, stored in the layers.

2) Parallel Processing: this refers to the fact that (a) the immune system is the composition of multiplecollaborative efforts of simple entities toward the same task. Moreover, (b) the simultaneous use of multiple (and therefore different) collaborative mechanisms for solving the same task may be useful. In an ensemble of autonomic components, just as in the 
TABLE I. CONNECTION BETWEEN COHEN IMMUNE SYSTEM AND BM'S MAPE-K.

\begin{tabular}{|l|l|}
\hline Cohen's Cognitive Model & MAPE-K feedback loop \\
\hline Body maintenance and protection & Managed resources \\
\hline Receptor and image repertoire & Knowledge \\
\hline Affinity between environmental signals and immune agents & Sensors \\
\hline Cognition & Monitor \\
\hline Recognition & Analyse \\
\hline Decision via co-respondence & Plan \\
\hline Inflammatory response & Execute \\
\hline Varying connectivity and concentration of immune agents & Effector \\
\hline
\end{tabular}

immune system, complex behaviour should emerge from the interactions of simple components.

3) Dynamic Engagement: in the biological immune system, cells that act to preserve the normal functioning of the body have short lives. They operate for short periods and then are replaced by similar cells. This implies that a response (plan of action) should be executed for a fixed period of time. In terms of an autonomic system, this implies that once an appropriate plan has been selected to go through the execution phase, an appropriate time for evaluating the ensemble performances should be allocated in order to avoid testing unsuitable solutions for unnecessarily long periods. On the other hand, one should also be aware that testing a potentially useful plan over a short period could lead to an un-representative evaluation.

4) Variable Connectivity: an immune network presents a non-linear topology of connected individuals that varies over time in both composition and connectivity. Once a pathogen is neutralized, local networks might quickly disappear, while other networks are persistent over time. By varying the connectivity of the network, the functionality of the network is modified. From the perspective of an autonomic system, a coordination pattern refers to a specific configuration of roles, interactions and behaviours of an ensemble. Changing the connectivity of the ensemble alters the network topology, and therefore may lead to changes in the mechanisms adopted to solve the specified task.

These principles lead to an obvious emergent behaviour of the immune system, i.e. the ability to adapt its response - in terms of the type of response and cells involved dynamically, to ensure that the most appropriate response is realised at any given time. This same phenomenon has been described in the autonomic computing literature by [20] who use the term self-expression and define it as the ability of an ensemble of autonomic components to change their coordination pattern during run time execution of tasks of variable complexity. Thus, we see an obvious parallel between a desired behaviour in a computational system and behaviour that occurs in the natural immune system. It should also be noted that immune system embodies another important concept with respect to self-adaptive autonomic systems - the need to be able to balance exploitation and exploration of behaviours to ensure the most appropriate response is chosen [21]. In the immune system exploration arises via somatic hypermutation processes that occur during cloning, while exploitation is achieved via germ-line encoding and memory. While maintaining an equivalent balance exploration and exploitation of coordination patterns of behaviour in an autonomic system is likely to be beneficial in leading to new adaptations, it should be noted that this has the potential to lead to a situation in which different components simultaneously follow different coordination patterns, thus introducing potential conflict. This may require additional design steps in order to prevent or deal with this consequence.

\section{EXAMPLE APPLICATION}

Having mapped out these concepts above, we briefly present an application in which these ideas have been implemented in a real-scenario in order to achieve self-expression in an autonomic system. We consider a simple foraging scenario implemented in a swarm of robots where the objective for nodes in the swarm is to select a suitable coordination pattern in order to maximise the amount of food found. Instead of providing a complete description of the coordination pattern implemented or a detailed discussion of the results obtained, we focus on describing the design process in connection with the concepts previously introduced. The interested reader is referred to [22] for a more detailed description.

In [22], we modelled a simple task in which a swarm of robots, initially randomly distributed in a confined arena, are required to collect food from a source and return that food to a nest, in an iterative process. The goal of the task is to maximise the total amount of food returned to the nest in a fixed time period. The robots we simulated do not have cognition of space nor orientation, hence we proposed multiple coordination patterns in order to solve the problem of creating a viable path from food to nest. We then designed an immune network in order to enable the ensemble to choose at run-time the best coordination pattern in order to solve this problem. Three coordination patterns are available.

The first pattern is a purely swarm approach, a robotic application of the ant colony optimization algorithm in which robots can act themselves as pheromones, so by lighting their LEDs with specific colours can underlines paths to be followed by the ant robots that are attracted to lights. This pheromone robots can dynamically expire so to try to optimize the length of this path.

The second coordination pattern is a robotic applications of a peer-to-peer $(\mathrm{p} 2 \mathrm{p})$ algorithm for distributing a gradient value that starts with its maximum value from the food area to then decrease when approaching the nest area. According to a communicative path formation algorithm similar to the ones used for networks or graphs (we have robots instead of nodes in this case), the robots will start by uniformly distributing throughout the arena and by applying this algorithm, a one robot-thick path from food area to nest will be created so that other robots may follow it. 
A baseline coordination effort is represented by a diffusive algorithm in which every robot always goes straight on until a collision is sensed: as a reaction the colliding robots will separate going though opposite directions, so to have the ensemble uniformly distributed throughout the whole arena.

We identify the same key concepts underlined in the previous section, mapped to this very specific problem:

- Managed resource: the ensemble and the environment. In particular the position of every single robot over time, their state and the amount of collected food.

- Sensor and effectors: mapped to sensors and actuators used by every robot.

- Knowledge: every robot is metaphorically a lymphnode, thus owning a set of interconnected antibodies with concentrations that can vary according to how the selected antibody performs over time. Each antibody (analogous to CBR related literature), is a tuple indicating conditions and corresponding actions to take. The conditions relate to the amount of food collected over a short period of time called evaluation time, sensed complexity of the arena (initial short-run experiments showed how the $\mathrm{p} 2 \mathrm{p}$ approach performs better in more complex arenas) and expected collected food over the evaluation time. The action field relates to the coordination pattern to be selected according to the sensed conditions.

- Monitor - Cognition: Obtaining external conditions though robotic sensors.

- Analyse - Recognition: Euclidean distance between the perceived environment and an antibody's condition field.

- Planning - Action selection: Select the highest concentrated antibody.

- Execute: Start to follow the coordination pattern determined by the action field of the previously selected antibody.

- Effector - Antibody dynamics: After the evaluation time, each robot is provided with positive feedback if the selected coordination pattern resulted in satisfying the expected amount of food collected after the evaluation time. Feedback is realised by increasing the affinity of all the other non-selected antibodies to the previously selected one. In case of negative feedback, the selected antibody will increase its affinity towards all the other antibodies. Affinity will be used to calculated the gradient over time of concentrations value of each single antibody.

- Group-Awareness: The highest concentrations of antibodies for each lymph node (robot) are now shared amongst other robots in range, in order to distribute knowledge, awareness and therefore experiences in subsets of the ensemble, thus trying to obtain a shared decision on the next coordination effort to undertake (see Fig. 3). The infra-red short range signalling communication among robots implies an ever varying connection topology over time as robots move throughout the arena.

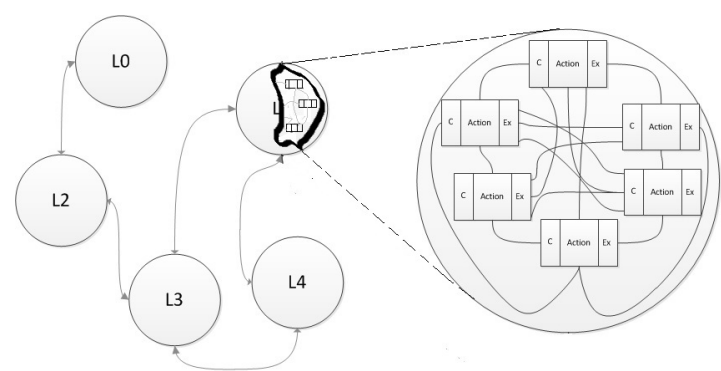

Fig. 3. lymph-nodes are robots with variable connectivity and they can host a multitude of interconnected antibodies.

With respect to the principles described by [9], we find the following mapping with the system just described:

- From an implementation point of view, it should be clear that all of these coordination patterns share a set of common component behaviours: for example, all contain a diffusion algorithm (active when there is no trail to follow), a mechanism for detecting points of interest (food, robots), and the ability to follow a gradient or trail. Each common behavioural component acts scaffolding for more than one coordination pattern. The components are retained in the network, enabling future use. The network of antibodies created inside each node sustains a collection of antibodies describing different situations, therefore the network is also able to retain knowledge. Thus, the Phylogenic Layering principle is present in the whole architecture.

- Through knowledge-sharing between robots, the system is potentially able to reach consensus on the coordination pattern to be used. However, the variable and unpredictable connectivity of the robot topology (due to the short range of the infra-red signalling devices) and diversity in the experiences among the different robots can cause the ensemble to create sub-sets of components, with each sub-set deploying different coordination patterns. We thus encapsulate Parallel Processing, with pleiotropic features in corespondence actions, i.e. component actions can be used in different coordination patterns, and a single single coordination pattern can be relevant in more than one situation.

- The dynamic engagement principle is symbolized by the fact that every decision taken by the ensemble is evaluated after a specific evaluation time, so as to avoid testing non-optimal coordination pattern for a disproportional length of time.

This whole approach was tested in three different arena configurations and evaluated in terms of total amount of collected food during the whole length of the experiment and amount of food collected over the evaluation time. Results presented in [22] show how the self-expression enables ensemble to develop learning, showing an increase in food collection over time, while this does not happen for the other coordination patterns evaluated in isolation. We believe this is a further proof of the emerging cognitive feature of this design immune net. Moreover, if we take a look to the decisions taken by the different robots in the arena, some important 


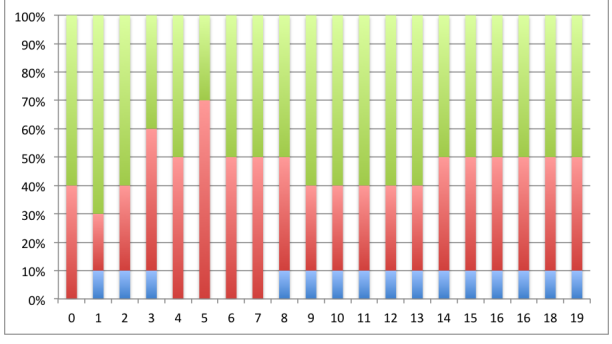

Fig. 4. The percentage of robots and their decision on coordination pattern over different time intervals. Red is for the $\mathrm{p} 2 \mathrm{p}$ approach, green for the swarm approach and in light blue the baseline diffusive coordination pattern.

remarks can be drawn: in Fig. 4 we can see how the ensemble autonomously decided to split into two big clusters, using two different coordination patterns. A small percentage of the ensemble decided to follow the baseline approach, either as a result of having obtained poor performances in the past with the other two patterns, or because of prolonged isolation from the rest of the group. This is actually helping the ensemble as a whole in reaching adaptivity: this small set of robots is actually maximizing the exploration concept in accordance to the exploration and exploitation principle.

\section{CONCLUSiON}

In this article we have considered the relationship between the concepts of awareness and adaptivity. In particular, we examined two design mechanisms for enabling Self-* properties - one from the autonomic computing literature and one from biology. Awareness in both these systems emerges as the systems are able to undertake decision-making and to learn from previous experiences. We also showed how these concepts can be used in ensembles of autonomic components and how the similarities between the IBM's MAPE-K control loop and Cohen's cognitive immune net can indeed help the designer taking advantage of these kind of decentralized, highly scalable architectures. As an example, a previously investigated scenario was exploited by highlighting how the design mechanisms have been taken into account.

For future work, all of this should be used for proposing a single merged framework for adaptation able to enrich the more traditional blueprints for feedback loops with the mechanisms able to deploy adaptivity that are inherit by AISinspired approaches.

\section{ACKNOWLEDGMENT}

The work is partially supported by the ASCENS project (EU FP7-FET, Contract No. 257414)

\section{REFERENCES}

[1] C. Humphrey and J. Adams, "Robotic tasks for chemical, biological, radiological, nuclear and explosive incident response," in RSJ Advanced Robotics: special issue on Disaster Response Robotics. Taylor and Francis, vol 23, Issue 9, 2009.

[2] R. Murphy, "Rescue robotics for homeland security," in Communications of the ACM - Homeland security. ACM, vol 47, Issue 3, March 2004.
[3] M. Viroli, G. Castelli, M. Mamei, and F. Zambonelli, "A coordination approach to adaptive pervasive service ecosystems," in Self-Adaptive and Self-Organizing Systems Workshops (SASOW), 2011 Fifth IEEE Conference on. Ann Arbor, Michigan, USA: IEEE Computer Society, October 2011, pp. 114-119.

[4] N. Capodieci, G. Cabri, A. Pagani, and M. Aiello, "An agent-based application to enable deregulated energy markets," in proceedings of The 36th International Conference on Computer Software and Applications (COMPSAC 2012). IEEE, 2012.

[5] J. Kephart and D. Chess, "The vision of autonomic computing," Computer, vol. 36, no. 1, pp. 41-50, 2003.

[6] L. de Castro and J. Timmis, "Artificial immune system: A new computational intelligence approach." Springer, 2002.

[7] I. Cohen, "Discrimination and dialogue in the immune system," in Seminars in immunology, 2000, pp. 215-219.

[8] P. Horn, "Autonomic computing: Ibm's perspective on the state of information technology," in Technical Report. IBM press, 2001.

[9] C. Ororsz, "An introduction to immuno-ecology and immunoinformatics," in Design Principles for the immune System and Other Distributed Systems, Edited by Segel and Cohen. Oxford Press, 2001.

[10] S. Stepney, "Embodiment," in In silico immunology, Edited by Flower and Timmis. Springer, 2006.

[11] P. Lewis, A. Chandra, and E. Robinson, "A survey of self-awareness and its application in computing systems," in 2011 Fifth IEEE Conference on Self-Adaptive and Self-Organizing Systems Workshops. Ann Arbor, Michigan, USA: IEEE Computer Society, October 2011.

[12] J. Schaumeier, J. Pitt, and G. Cabri, "A tripartite analytic framework for characterising awareness and self-awareness in autonomic systems research," in 2012 Sixth IEEE Conference on Self-Adaptive and SelfOrganizing Systems Workshops. Lyon, France: IEEE Computer Society, September 2012.

[13] N. Capodieci and G. Cabri, "Conceptual map and classification in ensembles of autonomic components: from awareness to organization," in 2012 Sixth IEEE Conference on Self-Adaptive and Self-Organizing Systems Workshops. Lyon, France: IEEE Computer Society, September 2012.

[14] H. Zhu, M. Hou, and M. Zhu, "Establishing the fundations of adaptive collaboration," in in Proc. of the ACM/IEEE International Symposium on Collaborative Technologies and Systems (CTS'10). Chicago, IL, USA: IEEE Computer Society, May 2010, pp. 546-554.

[15] R. Bruni, A. Corradini, F. Gadducci, A. Lluch-Lafuente, and A. Vandin, "A conceptual framework for adaptation." in FASE. LNCS 7212, Springer (2012) pp. 240-254. Springer, 2012.

[16] G. Cabri, M. Puviani, and F. Zambonelli, "Towards a taxonomy of adaptive agent-based collaboration patterns for autonomic service ensembles." in Collaborative Technologies and Systems, 2011 IEEE Conference on. Philadelphia, USA: IEEE Computer Society, May 2011.

[17] M. Jerne, "Towards a network theory of the immune system. annals of immunology," in Annals of immunology, 2 d ed. Cambridge, 1974, pp. 373-389.

[18] J. Hunt, D. Cooke, and H. Holstein, "Case memory and retrieval based on the immune system," in I ICCBR, Lecture Notes in Artificial intelligence 1010. Springer, 1995, pp. 205-216.

[19] I. Cohen, "Tending adam's garden: evolving the cognitive immune self," in Elsevier Academic Press, 2004.

[20] F. Zambonelli, N. Bicocchi, G. Cabri, L. Leonardi, and M. Puviani, "On self-adaptation, self-expression, and self-awareness, in autonomic service component ensembles," in Self-Adaptive and Self-Organizing Systems Workshops (SASOW), 2011 Fifth IEEE Conference on. Ann Arbor, Michigan, USA: IEEE Computer Society, October 2011, pp. 108-113.

[21] J. Holland, "Adaptation in natural and artificial systems," in $2 d$ ed. Cambridge,, MA: MIT Press, 1992.

[22] N. Capodieci, E. Hart, and G. Cabri, "An immune network approach for self-adaptive ensembles of autonomic components: a casestudy in swarm robotics," in Proceedings of the 12th European Conference on Artificial Life (ECAL), 9 2013. [Online]. Available: http://www.dmi.unict.it/ecal2013/ 